The electronic and transport properties of a molecular junction studied by an integrated piecewise thermal equilibrium approach

M.-H. Tsai, T.-H. Lu, and Y.-H. Tang

Citation: Journal of Applied Physics 104, 043703 (2008); doi: 10.1063/1.2970164

View online: https://doi.org/10.1063/1.2970164

View Table of Contents: http://aip.scitation.org/toc/jap/104/4

Published by the American Institute of Physics

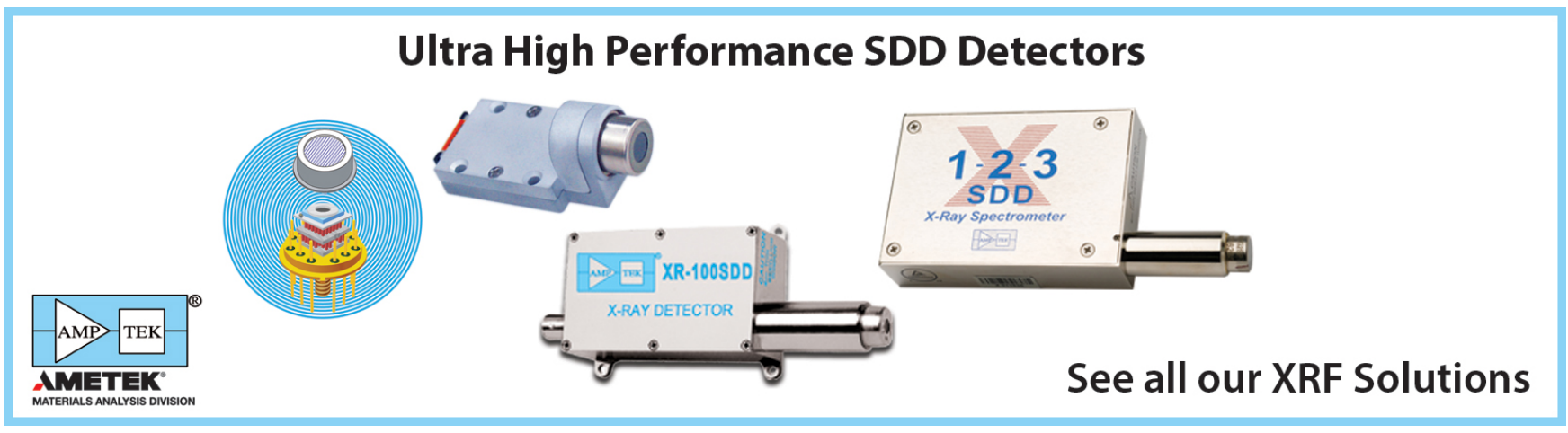




\title{
The electronic and transport properties of a molecular junction studied by an integrated piecewise thermal equilibrium approach
}

\author{
M.-H. Tsai, ${ }^{\text {a) }}$ T.-H. Lu, and Y.-H. Tang ${ }^{\text {b) }}$ \\ Department of Physics, National Sun Yat-Sen University, Kaohsiung 80424, Taiwan
}

(Received 7 February 2008; accepted 20 June 2008; published online 25 August 2008)

\begin{abstract}
An integrated piecewise thermal equilibrium approach based on the first-principles calculation method has been developed to calculate bias dependent electronic structures and current- and differential conductance-voltage characteristics of the gold-benzene-1,4-dithiol-gold molecular junction. The calculated currents and differential conductance have the same order of magnitude as experimental ones. An electron transfer was found between the two electrodes when a bias is applied, which renders the two electrodes to have different local electronic structures. It was also found that when $\mathrm{Au} 5 d$ electrons were treated as core electrons the calculated currents were overestimated, which can be understood as an underestimate of the Au-S covalent bonding and consequently the contact potential barrier and the replacement of delocalized Au $5 d$ carriers by more itinerant delocalized $\mathrm{Au} 6 s p$ carriers in the electrodes. (C) 2008 American Institute of Physics.
\end{abstract}

[DOI: $10.1063 / 1.2970164]$

\section{INTRODUCTION}

Studies of electron transport through organic molecules as the possible components in molecular electronics are of both fundamental and technological importance. Fabrication and measurements of current-voltage $(I-V)$ and/or conductance-voltage $(C-V)$ characteristics of metalmolecule-metal systems using various organic molecules have been achieved by Reed et al., ${ }^{1}$ Chen et al., ${ }^{2}$ Tian et al., Xiao et al. ${ }^{4}$ Dadosh et al., ${ }^{5}$ and Kiguchi et al. ${ }^{6}$ Among the various organic molecules considered, the benzene-1,4dithiol (BDT) molecule is the simplest. The experimentally observed transport properties of these metal-molecule-metal systems have attracted intensive theoretical investigations. ${ }^{7-39}$ Nitzan and Ratner ${ }^{40}$ have reviewed the research on the electron transport in molecular wire junctions.

Theoretical calculations of the electric current $I$ have been commonly based on the standard expression

$$
I=\frac{2 e}{\hbar} \int_{-\infty}^{\infty} T(E)\left[f\left(E-\mu_{1}\right)-f\left(E-\mu_{2}\right)\right] d E,
$$

where $f$ is the Fermi-Dirac distribution function, $\mu_{1}$ and $\mu_{2}$ are the bias dependent chemical potentials at the two metal electrodes, and $T(E)$ is the transmission coefficient obtained by, for example, the scattering theory of transport ${ }^{41}$ and nonequilibrium Green function (NEGF) approach. ${ }^{42-46}$ The NEGF formalism of Keldysh, Kadanorf, and Baym has usually been employed in conjunction with the density functional theory (DFT)-based first-principles calculation methods to calculate transport properties of molecular junctions. For example, Xue and Ratner $^{28}$ carried out a microscopic study of end group effect on electrical transport through individual molecules, Kondo et $a .^{29}$ studied the contact-

\footnotetext{
${ }^{a)}$ Author to whom correspondence should be addressed. Electronic mail: tsaimh@mail.nsysu.edu.tw.

b) Present address: Department of Physics, California State University, Northridge, CA 91330.
}

structure dependence of transport properties of a single BDT molecule between $\mathrm{Au}(111)$ electrodes, Chen et al. ${ }^{30}$ studied the control of substituent ligand over current through molecular devices, and Waldron et al. ${ }^{31}$ studied nonlinear spin current and magnetoresistance of a Ni-BDT-Ni molecular magnetic tunnel junction. On the other hand, Geng et al. ${ }^{32}$ used the Lippmann-Schwinger scattering method in conjunction with DFT and studied the impact of metal electrode and molecular orientation on the conductance of a single BDT molecule.

In the scattering theory and NEGF approach, the molecular junction is usually divided into three parts, namely, two semi-infinite electrodes and an "extended molecule," which includes BDT and a part of the electrode in contact or bonded with the molecule. The two semi-infinite electrodes were regarded as reservoirs of itinerant carriers. In the NEGF/DFT approach, the effect of bias is manifested in the external potential in the Kohn-Sham equation. The molecule-electrode interaction is manifested as the selfenergy. Brandbyge et al. ${ }^{44}$ found a correspondence between the transfer matrices derived from the scattering theory and NEGF. Faleev et al. $^{33}$ found that the use of NEGF reduced the calculated current by about one-half from that obtained by a zero-bias transmission coefficient at a bias of $4 \mathrm{~V}$ for the gold-BDT-gold molecular junction.

In previous theoretical studies, the molecule is usually regarded as chemisorbed at the hollow site on the flat surfaces of the Au electrodes. In this chemisorption picture, the $\mathrm{S}$ atom is bonded with multiple $\mathrm{Au}$ atoms. Despite intensive efforts of previous theoretical investigations, the calculated currents for the gold-BDT-gold junction were persistently one to three orders of magnitude larger than the experimental data of Reed et al., ${ }^{1}$ except the ones by Wang and Lou ${ }^{15}$ and Wang et al., ${ }^{16}$ who used the hybrid DFT of quantum chemistry. When Di Ventra et al. ${ }^{24}$ considered that the $\mathrm{S}$ end of BDT is attached to the flat jellium surface, the calculated currents were about 500 times larger than experimental data 
of Reed et al. ${ }^{1}$ However, when they considered the case that each $\mathrm{S}$ end is bonded with a single Au atom and the single $\mathrm{Au}$ atom is then attached to the flat jellium surface, the calculated currents were greatly improved and reduced down to only about one order of magnitude larger than experimental data. A similar contact geometry, in which each $\mathrm{S}$ end of BDT is bonded with a single Au atom, was also considered by Cuevas et al. ${ }^{35}$

Guided by the finding of Di Ventra et al. ${ }^{24}$ the geometry of the molecule-electrode contact in this study is chosen such that each $\mathrm{S}$ end of BDT is bonded with a single Au atom and this single $\mathrm{Au}$ atom is then chemisorbed at the threefold hollow site of the $\mathrm{Au}(111)$ surface. For the first-principles calculation method to be used in this study, the charge density and potential in the interstitial region, which includes the electrode-molecule interface regions, are described by plane waves; it is difficult to derive the open boundary conditions (self-energy) at the molecular junction. To avoid the complexity of dividing the molecular junction into three parts, namely, two electrodes and an extended molecule, and corresponding division of interactions, potentials, and Hamiltonian matrix, an integrated piecewise thermal equilibrium approach has been developed and tested on the gold-BDTgold molecular junction. The calculated currents and conductance have the same order of magnitudes as those obtained by Reed et al. ${ }^{1}$ For example, the current per molecule obtained in this study is $0.050 \mu \mathrm{A}$ at a bias of $1.5 \mathrm{~V}$ versus $\sim 0.03 \mu \mathrm{A}$ obtained by Reed et al. ${ }^{1}$ at the same bias. Also zero-bias conductance obtained in this study is $0.0062 \mu \mathrm{S}$ while the experimental value of Reed et al. ${ }^{1}$ was $\sim 0.002 \mu \mathrm{S}$.

\section{THE STRUCTURAL MODEL AND THE FIRST- PRINCIPLES METHODS USED}

There are two kinds of structural models used for studying surfaces. One is the semi-infinite model, which contains only one surface. Another is the slab model, which includes the single slab model with (in principle) semi-infinite vacuum regions on both sides and infinitely repeated-slab model or supercell model usually used in pseudopotential calculations. The slab model has been widely employed in first-principles studies of surface science. In this study the single slab model is chosen for the gold electrode. Due to the screening effect of conduction electrons, a three-atomic-layer slab already has a total DOS, D $(E)$, similar to that of the bulk crystal of metals. Thus, a three-layer $\mathrm{Au}(111)$ slab model is chosen. An extension to a thicker-slab model can be considered later. A schematic drawing of the optimized Auatom-BDT-Au-atom molecule and the gold slabs is shown in Fig. 1. The Au atom bonded with the $\mathrm{S}$ atom is located at the threefold hollow site above the $\mathrm{Au}(111)$ film surface. The plane of the benzene ring is parallel with the ${ }^{1}$ basis vector of the $\mathrm{Au}(111)$ film. $\mathrm{A}(3 \times 2) \mathrm{Au}(111)$ periodicity is chosen for the self-assembled monolayer (SAM) of BDT molecules so that the unit cell has totally $38 \mathrm{Au}, 2 \mathrm{~S}, 6 \mathrm{C}$, and $4 \mathrm{H}$ atoms. This unit cell is chosen to avoid direct overlapping among BDT molecules. The periodicity of the $\mathrm{Au}(111)$ film is $2.885 \AA$, so the two-dimensional unit cell is 8.655

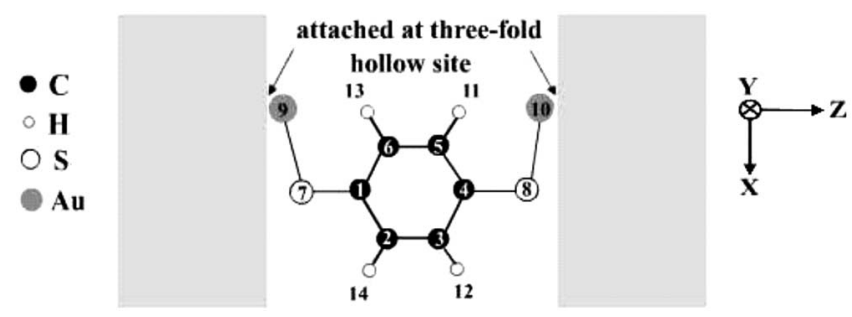

FIG. 1. A schematic drawing of the gold-SAM-gold trilayer system.

$\times 4.997 \AA^{2}$. The atomic positions of the BDT molecule have been optimized by the first-principles molecular dynamics (MD) calculation method. ${ }^{47,48}$ The MD method is based on the real-space norm-conserving pseudopotential method ${ }^{49,50}$ implemented with the Ceperley-Alder exchange-correlation potentials. ${ }^{51}$ The basis set is composed of Bloch sums of $s$, $p_{x}, p_{y}$, and $p_{z}$ pseudoatom orbitals. This method efficiently calculates the forces on atoms using the modified HellmannFeynman theorem, ${ }^{52}$ which are then used to predict the equilibrium atomic positions iteratively until the magnitudes of the forces are less than $0.1 \mathrm{eV} / \AA$. The modified pseudofunction (PSF) method ${ }^{53,54}$ implemented with the linear theory of Andersen ${ }^{55}$ is used to calculate the electronic structure and the total energy. The basis set used in the PSF method contains two sets of Bloch sums of muffin-tin orbitals with exponentially decaying spherical Hankel functions, which describe localized lower-energy states, and oscillating Neumann tailing functions, which describe higher-energy conduction-band states. Inside the muffin-tin sphere, the muffin-tin orbital is a linear combination of the solution of the atomic Kohn-Sham equation and its energy derivative within the linear theory of Andersen. ${ }^{55}$ The muffin-tin orbital and its radial derivative are continuous at the muffin-tin radius. The PSFs are constructed by replacing muffin-tinsphere parts of the muffin-tin orbitals by smooth mathematical functions so that their Bloch sums are expandable by a small number of plane waves; they are simply devised to calculate the interstitial and nonspherical parts of the Hamiltonian matrix elements efficiently using the fast-Fouriertransform (FFT) technique. The PSF method uses the Hedin-Lundqvist ${ }^{56}$ form of local density approximation (LDA) for the exchange-correlation potential. The four special $\vec{k}$ point scheme of Cunningham ${ }^{57}$ is used to obtain selfconsistent charge densities and potentials.

The average $\mathrm{C}-\mathrm{C}, \mathrm{C}-\mathrm{H}$, and $\mathrm{C}-\mathrm{S}$ bond lengths obtained in this study are $1.428,1.159$, and $1.929 \AA$, respectively, which differ from the experimental $\mathrm{C}-\mathrm{C}$ and $\mathrm{C}-\mathrm{H}$ bond lengths in a benzene molecule of 1.399 and $1.101 \AA$ (Ref. 58 ) by $2.07 \%$ and $5.26 \%$, respectively, and the $\mathrm{C}-\mathrm{S}$ singlebond length in $\left(\mathrm{CH}_{2}\right)_{2} \mathrm{~S}\left(\mathrm{CH}_{2}\right)_{2}$ of $1.839 \AA$ (Ref. 58) by $4.89 \%$. The $\mathrm{C}-\mathrm{S}$ bond lengths obtained by Faleev et al. ${ }^{33}$ and Stokbro et al. ${ }^{19}$ were 1.74 and $1.75 \AA$, respectively. The calculation of the total energies with respect to the adjustment of the $\mathrm{Au}-\mathrm{S}$ distance and the $\mathrm{Au}-\mathrm{S}-\mathrm{C}$ angle yields a $\mathrm{Au}-\mathrm{S}$ bond length of $2.20 \AA$, which is about $7 \%$ smaller than $2.42,{ }^{12} 2.39,{ }^{19}$ and $2.37 \AA$ (Ref. 35) obtained previously for the $\mathrm{S}$ atom coordinated with multiple $\mathrm{Au}$ atoms, and a $\mathrm{Au}-$ $\mathrm{S}-\mathrm{C}$ bond angle of $98.9^{\circ}$, which is within the range of known 
bond angles of $\mathrm{S}$; for example, $\angle \mathrm{F}-\mathrm{S}-\mathrm{F}=98.2^{\circ}$ in $\mathrm{SF}_{2}$, $\angle \mathrm{Cl}-\mathrm{S}-\mathrm{Cl}=103^{\circ}$ in $\mathrm{SCl}_{2}$, and $\angle \mathrm{C}-\mathrm{S}-\mathrm{H}=96.4^{\circ}$ in $\mathrm{CH}_{3} \mathrm{CH}_{5}-\mathrm{SH}^{58}$ Chen et al. ${ }^{30}$ obtained a $\mathrm{Au}-\mathrm{S}$ bond length of $2.37 \AA$ and a Au-S-C bond angle of $102.8^{\circ}$ for the $\mathrm{Au}-$ atom-BDT-Au-atom molecule. In comparison, Yourdshahyan et al. $^{59}$ obtained a much larger $\mathrm{Au}-\mathrm{S}-\mathrm{C}$ bond angle between $132^{\circ}$ and $138^{\circ}$ for the long-chain alkane thiols $\left[\mathrm{CH}_{3}\left(\mathrm{CH}_{2}\right)_{n-1} \mathrm{SH}\right]$ chemisorbed on the $\mathrm{Au}(111)$ surface. The calculated partial densities of states (PDOSs) of S and C atoms and the $\mathrm{Au}$ atoms that are bonded with the $\mathrm{S}$ atoms are shown in Fig. 2, in which the Fermi level $E_{F}$ is chosen as the zero energy. Figure 2 shows that there is a band gap of 0.77 $\mathrm{eV}$ and that the highest occupied molecular orbital (HOMO) energy levels are composed of S $3 p, \mathrm{C} 2 p$, and Au $5 d$ hybridized states while the lowest unoccupied molecular orbital (LUMO) energy levels are composed of $\mathrm{S} 3 p, \mathrm{C} 2 p$, $\mathrm{Au} 6 s p$, and $5 d$ hybridized states. The wide spreading of the $\mathrm{Au} 5 d$ states and the overlapping of PDOSs of $\mathrm{Au} 5 d$ and S $3 p$ states indicate a significant part of covalent bonding between $\mathrm{Au}$ and $\mathrm{S}$ via $\mathrm{Au} 5 d$ orbitals.

\section{THE INTEGRATED PIECEWISE THERMAL EQUILIBRIUM APPROACH}

In this approach, the nonequilibrium distribution function of electrons in the metal-SAM-metal trilayer system with a bias applied to the two metal electrodes is approximated by a piecewise thermal equilibrium distribution function. This is an adequate approximation because electrons can respond fast enough to the local potential similar to the success of the LDA for the exchange-correlation energy, which approximates the nonuniform electron density as piecewise uniformly distributed. With this approximation the

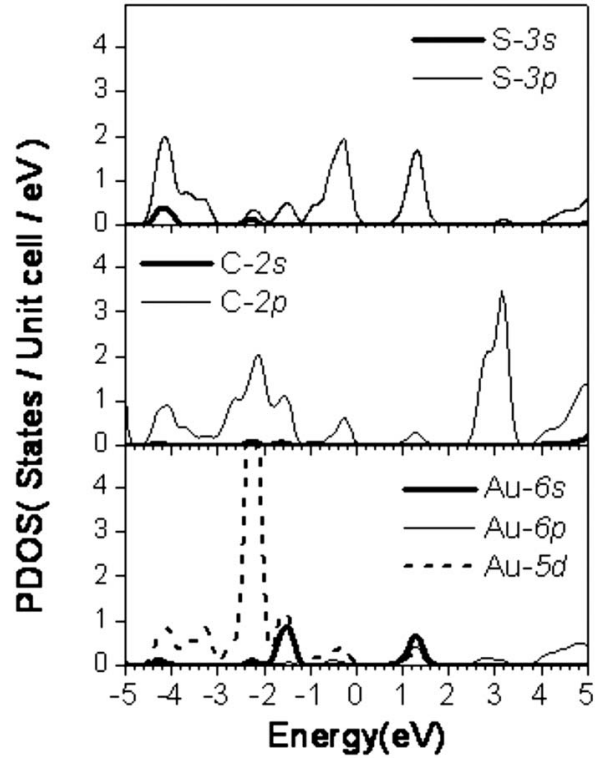

FIG. 2. PDOSs of the SAM of Au-BDT-Au molecules (without Au electrodes). The peak value of the $\mathrm{Au} 5 d$ feature located at $-2.3 \mathrm{eV}$ is 8.0 states/unit cell/eV.

nonequilibrium distribution function of electrons is given as $f[E, T, \mu(z)]=\left\{1+\exp \left[(E-\mu(z)) /\left(k_{B} T\right)\right]\right\}^{-1}$, where $E, T$, $\mu(z)$, and $k_{B}$ are the eigenenergy, absolute temperature, local chemical potential, and Boltzmann's constant, respectively. The z-coordinate is perpendicular to the trilayer system. Since the resistivity of the molecule is much higher than that of the electrodes, the potential drop due to the bias is expected to be entirely in the molecule as already shown by Kondo et al. ${ }^{29}$ and Faleev et al. ${ }^{33}$ Thus, the $z$-dependent local chemical potential is approximated as

$$
\mu(z)= \begin{cases}\frac{\mu_{1}+\mu_{2}}{2}+\frac{\left(\mu_{2}-\mu_{1}\right)}{2} \cos \left[\frac{\pi(z-b+2 d)}{2(d-b)}\right], & -d<z<-b \\ \mu_{1}, & -b \leq z \leq-t \\ \mu_{1}+\frac{\left(\mu_{2}-\mu_{1}\right)}{2 t}(z+t), & t \leq z<t \\ \mu_{2}, & t \leq z \leq b \\ \frac{\mu_{1}+\mu_{2}}{2}+\frac{\left(\mu_{2}-\mu_{1}\right)}{2} \cos \left[\frac{\pi(z-b)}{2(d-b)}\right], & b<z \leq d,\end{cases}
$$

where $\mu_{1}$ and $\mu_{2}$ are the constant chemical potentials in the two metal slabs within $(-b,-t)$ and $(t, b)$, respectively, and $\mu(z)$ drops linearly from $\mu_{1}$ to $\mu_{2}$ in the Au-atom-BDT-Auatom molecule between $-t$ and $t$. The bias voltage $V_{\text {bias }}$ is related to $\mu_{1}$ and $\mu_{2}$ by $(-e) V_{\text {bias }}=\mu_{2}-\mu_{1}$. The parts of $\mu(z)$ outside the trilayer system between $(-d,-b)$ and $(b, d)$ are smooth mathematical functions extended beyond $z=-b$ and $z=b$ with $\mu(-d)=\mu(d)$, which are chosen such that $\mu(z)$ is continuous and differentiable at $z= \pm b$ so that $\mu(z)$ can be accurately expanded by a minimal number of plane waves with wave numbers of $2 \pi m /(2 d)$, where $m$ is an integer, in order to be easily implemented in the PSF method. Note that plane wave expansion with wave numbers $2 \pi m /(2 d)$ in fact is a periodic function with a period of $2 d$. If $\mu(-d)$ is not equal to $\mu(d)$, there is a discontinuity of $\mu(z)$ at $z=d$ and $z$ $=-d$. In this case, a large number of plane waves will be needed to accurately describe $\mu(z)$. It has been tested that with $d$ and $b$ of 14.40 and $12.34 \AA$, respectively, and $|m|$ 
$\leqq 34 \mu(z)$ can be accurately represented by the plane wave expansion. In this study $\mu(z)$ is fixed, which can be determined self-consistently later. The external potential $v_{\text {ext }}(z)$ added to the effective potential in the Kohn-Sham equation is given by $\nu_{\text {ext }}(z)=\mu(z)-\Delta \nu_{\text {Coul }}$. The Coulomb potential difference across the trilayer system, $\Delta \nu_{\text {Coul }}$, is calculated as $\Delta \nu_{\text {Coul }}=\left\langle\nu_{\text {Coul }}(x, y, b)\right\rangle-\left\langle\nu_{\text {Coul }}(x, y,-b)\right\rangle$, where \langle\rangle stands for the average over the $(x, y)$ plane and $\nu_{\text {Coul }}(x, y, z)$ is the Coulomb potential calculated from the total electron charge density and ion core charges. Both $\nu_{\text {ext }}(z)$ and $\nu_{\text {Coul }}(x, y, z)$ are determined self-consistently. The subtraction of $\Delta \nu_{\text {Coul }}$ ensures that the difference of the total Coulomb potential of the system, $\nu_{\text {ext }}(z)+\nu_{\text {Coul }}(x, y, z)$, between the outside surfaces of the two slabs is equal to $(-e) V_{\text {bias }}$.

If $E_{n, \vec{k}}$ and $\Psi_{n, \vec{k}}(x, y, z)$ are the $n$th eigenenergy and eigenfunction of the Kohn-Sham equation of the whole trilayer system under the external potential $v_{\text {ext }}(z)$, the occupation number of this state, $f_{n, \vec{k}}$, is calculated as the expectation value of the Fermi-Dirac distribution operator, i.e.,

$$
f_{n, \vec{k}}=\left\langle\Psi_{n, \vec{k}}\left|f\left[E_{n, \vec{k}}, T, \mu(z)\right]\right| \Psi_{n, \vec{k}}\right\rangle .
$$

The electron density, $n(x, y, z)$, is then calculated as

$$
n(x, y, z)=2 \sum_{\vec{k}} \omega_{k}^{\vec{k}} \sum_{n} f_{n, k} \Psi_{n, \vec{k}}^{*}(x, y, z) \Psi_{n, \vec{k}}(x, y, z) .
$$

The factor 2 is due to spin degeneracy and $\omega_{k}^{-}$is the weighting factor of the special $\vec{k}$ point. As usual, $E_{n, \vec{k}}$ and $\Psi_{n, \vec{k}}(x, y, z)$ as well as $n(x, y, z)$ are determined selfconsistently. Within the PSF method, the Bloch sums of PSFs, $P_{l}^{k}(x, y, z)$, are identical to the basis wave functions, i.e., Bloch sums of muffin-tin orbitals in the interstitial region and are expanded as

$$
\begin{aligned}
P_{l}^{\vec{k}}(x, y, z)= & \sum_{\vec{G}_{/ /}} \sum_{G_{z}} P_{\vec{G}_{/ /}, l}^{\vec{k}}, \\
& \times \exp \left\{-i\left(\vec{G}_{/ /}-\vec{k}\right) \cdot(x \hat{i}+y \hat{j})\right\} \exp \left\{-i G_{z} z\right\},
\end{aligned}
$$

where $\vec{G}_{/ /}$is the two-dimensional reciprocal vectors and $G_{z}$ $=2 \pi m /(2 d)$ with $m=0, \pm 1, \pm 2, \pm 3, \ldots$ and $l$ is the index of the basis wave function in the basis set. The Bloch sums of PSFs can be readily decomposed into $+z$ - and $-z$-direction traveling waves. The two $+z$ and $-z$ traveling waves, $P_{l,+}^{\vec{k}}(x, y, z)$ and $P_{l,-}^{\vec{k}}(x, y, z)$, are constructed as

$$
\begin{aligned}
P_{l+}^{\vec{k}}(x, y, z)= & 1 / \sqrt{2} \sum_{\vec{G}_{/ /}} \sum_{G_{z}<0} P_{G_{/ /}}^{\vec{k}}, l \\
& \times \exp \left\{-i\left(\vec{G}_{/ /}-\vec{k}\right) \cdot(x \hat{i}+y \hat{j})\right\} \exp \left\{-i G_{z} z\right\}, \\
P_{l,-}^{\vec{k}}(x, y, z)= & 1 / \sqrt{2} \sum_{\vec{G}_{/ /}} \sum_{G_{z}>0} P_{\vec{G}_{/ /}}^{\vec{k}}, l \\
& \times \exp \left\{-i\left(\vec{G}_{/ /}-\vec{k}\right) \cdot(x \hat{i}+y \hat{j})\right\} \exp \left\{-i G_{z} z\right\},
\end{aligned}
$$

respectively. The factor $\frac{1}{\sqrt{2}}$ is to compensate for the split of one state, which is occupied with two electrons, into two states occupied with four electrons. Note that each Bloch sum is associated with a crystal momentum, $\hbar \vec{k}$, where $\vec{k}$ is a wave vector in the two-dimensional Brillouin zone. Thus, basis wave functions are traveling waves in the $(x, y)$ plane. However, they are not traveling waves in the $z$-direction. The decomposition of basis wave functions or Bloch sums of PSFs in the $z$-direction is similar to the decomposition of standing waves confined in a square box, say between $z$ $=-b$ and $z=b$, into $+z$ - and $-z$-direction traveling waves. For plane wave based first-principles methods such as the pseudopotential and linear augmented plane wave methods, the basis wave functions are already traveling waves so that decomposition is not needed.

If the eigenvector corresponding to the eigenvalue $E_{n, k}$ is $\left\{a_{n, l}^{k}\right\}$, the $+z$ and $-z$ traveling waves in the interstitial region are

$$
\begin{aligned}
\Psi_{n+}^{\vec{k}}(x, y, z)= & 1 / \sqrt{2} \sum_{l} a_{n, l}^{\vec{k}} \sum_{\vec{G}_{/ /}} \sum_{G_{z}<0} P_{\vec{G}_{/ /}, G_{z}, l}^{\vec{k}} \exp \left\{-i\left(\vec{G}_{/ /}-\vec{k}\right)\right. \\
& \times(x \hat{i}+y \hat{j})\} \exp \left\{-i G_{z} z\right\}, \\
\Psi_{n-}^{\vec{k}}(x, y, z)= & 1 / \sqrt{2} \sum_{l} a_{n, l}^{\vec{k}} \sum_{\vec{G}_{/ /}} \sum_{G_{z}>0} P_{\vec{G}_{/ /}, G_{z}, l}^{\vec{k}} \exp \left\{-i\left(\vec{G}_{/ /}-\vec{k}\right)\right. \\
& \times(x \hat{i}+y \hat{j})\} \exp \left\{-i G_{z} z\right\} .
\end{aligned}
$$

The total current densities in the interstitial region moving in $+z$ - and $-z$-directions, $j_{ \pm}$, can be expressed as

$$
\begin{aligned}
j_{ \pm}(x, y, z)= & \frac{i e \hbar}{m_{e}} \sum_{\vec{k}} \omega_{k}^{-} \sum_{n} f_{n, \vec{k}}\left[\Psi_{n \pm}^{k^{*}}\left(\frac{\partial}{\partial z} \Psi_{n \pm}^{\vec{k}}\right)\right. \\
& \left.-\left(\frac{\partial}{\partial z} \Psi_{n \pm}^{\vec{k}^{*}}\right) \Psi_{n \pm}^{\vec{k}}\right],
\end{aligned}
$$

by applying the standard quantum mechanical current density equation, $\vec{j}=\frac{i e \hbar}{2 m_{e}}\left[\Psi^{*}(\nabla \Psi)-\left(\nabla \Psi^{*}\right) \Psi\right]$, for the electron with a charge $(-e)$, where $m_{e}$ is the electron mass. These two current densities can be efficiently calculated by FFT and expressed as

$$
\begin{aligned}
j_{ \pm}(x, y, z)= & \sum_{\vec{G}_{/ /}} \sum_{G_{z}} J_{G_{/ /}, G_{z}, \pm} \exp \left\{-i \vec{G}_{/ /} \cdot(x \hat{i}+y \hat{j})\right\} \\
& \times \exp \left\{-i G_{z} z\right\} .
\end{aligned}
$$

The $(x, y)$ plane averaged current densities are given by

$$
j_{ \pm}(z)=\sum_{G_{z}} J_{0, G_{z}, \pm} \exp \left\{-i G_{z} z\right\} .
$$

The current through the molecule is calculated as $I$ $=A\left[j_{+}\left(z_{m}\right)+j_{-}\left(-z_{m}\right)\right]$, where $A$ is the area of the unit cell, which contains one molecule, and $z_{m}$ is the end position of the molecule, at which PSFs are identical to real basis wave functions in $z= \pm z_{m}(x, y)$ planes. Since the trilayer structural model is not a closed circuit, the current $I^{\prime}=A\left[j_{-}\left(z_{m}\right)\right.$ $\left.+j_{+}\left(-z_{m}\right)\right]$ compensates for $I$ so that there is no net current flow. However, in the experimental current measurements, a closed circuit has to be connected. For example, the two gold electrodes are connected to a battery or a power supply. The current $I^{\prime}$ will be drained into the battery or power supply. 
Then, there will be a net current $I$ flow through the molecule. The present approach mimics the drift current, which is the difference between two opposite-direction currents due to traveling waves moving in opposite directions, in a finitesized section of metal.

The traveling wave formalism is conceptually the same as the calculation of the transmission probability of a particle passing through a one-dimensional (1D) potential barrier/ well given in most quantum mechanics textbooks. The $A j_{+}\left(z_{m}\right)$ and $A j_{-}\left(-z_{m}\right)$ currents are analogous to $(2 e / \hbar) \int_{-\infty}^{\infty} T(E) f\left(E-\mu_{1}\right) d E$ and $-(2 e / \hbar) \int_{-\infty}^{\infty} T(E) f\left(E-\mu_{2}\right) d E$ currents given in Eq. (1). The former corresponds to the current due to traveling waves that impinge from the left $(-z)$ side and pass through the potential barrier/well or the molecule to reach the right $(+z)$ side. The latter corresponds to the current in the opposite direction and the net current is the difference between them. The major difference between present formalism and those based on the scattering theory and NEGF approach is that in the present case the $+z$-direction $(-z$-direction) traveling waves at the $+z(-z)$ end of the molecule are solved directly from the Hamiltonian of the whole system just like the transmitted traveling waves are solved in the 1D potential barrier/well problem given in standard quantum mechanics textbooks. In contrast, the whole electrode-molecule-electrode system is divided into two electrodes and one extended-molecule part and $T(E)$ is calculated using the Lippmann-Schwinger equation or the correlation (lesser Green) function in previous calculations.

The difference with the 1D potential barrier/well case is that in the present case the slabs are terminated by vacuum. The effect of the slab-vacuum interface can be reduced by considering a thicker-slab model. Recently, Zhang et al. ${ }^{60}$ embedded an absorbing layer in the slab of an infinitely repeated slab-molecule-slab-vacuum supercell model and derived a three-dimensional generalized Bloch theorem to calculate quantum transport of a molecular junction. The absorbing layer was devised to simulate a reservoir, which drains the current. However, the current in a closed electric circuit does not flow in this way. For any section in a closed circuit, if the current drains from one end of this section, in the steady state there must be a current source that supplies the same current to the other end of this section. Thus, the absorption-layer model is not considered in the present study.

\section{ELECTRONIC STRUCTURES OF THE GOLD- SAM-GOLD TRILAYER SYSTEM}

Figure 3 shows the calculated PDOSs of (a) Au atoms and (b) S, C, and $\mathrm{H}$ atoms of the gold-SAM-gold trilayer system at $V_{\text {bias }}=0$. The calculated PDOSs of the bulk gold crystal are also shown in the top panel of Fig. 3(a) to elucidate the contact effect and to show the adequacy of the threelayer slab model for the electrode. In these figures, the constant chemical potential or Fermi level $E_{F}$ is chosen as zero energy. The top panel shows that the leading edge of the major $\mathrm{Au} 5 d$ features is located at $\sim 1.0 \mathrm{eV}$ below $E_{F}$. In the vicinity of $E_{F}$, the states are mainly Au $6 s p$ states. The Au $6 s$ and $6 p$ bands spread over a very large energy range, which indicates that delocalized Au $6 s$ and $6 p$ electrons are
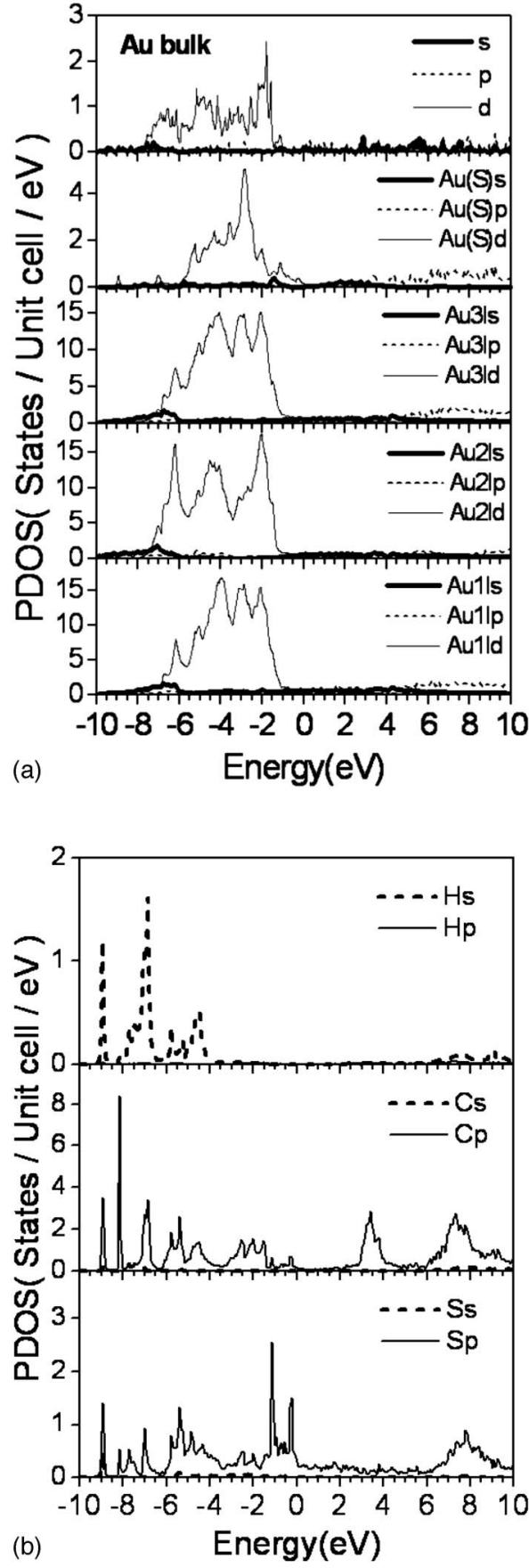

FIG. 3. (a) PDOSs of Au atoms in first, second, and third layers and the one bonded with the $\mathrm{S}$ atom denoted as $\mathrm{Au}(\mathrm{s})$ and (b) of $\mathrm{S}, \mathrm{C}$, and $\mathrm{H}$ in the zero-bias gold-SAM-gold trilayer system.

highly mobile. The Au $5 d$ band spreads with a band width of about $6.5 \mathrm{eV}$. For the $V_{\text {bias }}=0$ gold-SAM-gold trilayer system, the overall width of the major $5 d$ features of the $\mathrm{Au}$ atoms in the two slabs is about $6.5 \mathrm{eV}$, which is the same as the $5 d$ band width of the bulk gold crystal. However, the PDOSs of these $\mathrm{Au} 5 d$ bands do not drop to zero at $-1.0 \mathrm{eV}$, but tail off gradually up to about $8 \mathrm{eV}$ above $E_{F}$, which indicates that a small percentage of $\mathrm{Au} 5 d$ states is highly delocalized. The overall width of the major $5 d$ features of the single $\mathrm{Au}$ atom bonded with the $\mathrm{S}$ atom of BDT is about $6.0 \mathrm{eV}$, which is narrower than that of $\mathrm{Au}$ atoms in the slabs. The major $5 d$ features of this single Au atom shift 
to higher energies with the leading edge extending above $E_{F}$ due to bonding with the $\mathrm{S}$ atom. Similar to the bulk gold crystal, the Au $6 s$ and $6 p$ bands spread over a very large energy region. In the vicinity of $E_{F}$, PDOSs of Au $6 s, 6 p$, and $5 d$ states are approximately equal. The result that the overall width of the major $\mathrm{Au} 5 d$ features in the central layer [denoted by Au 21 in Fig. 3(a)] of the slab is the same as that of the bulk gold crystal and the shapes of $5 d, 6 s$, and $6 p$ bands roughly resemble those of the bulk gold crystal, which indicates that the three-layer slab model considered in this study for the gold electrode is adequate. The PDOSs of S and $\mathrm{C}$ atoms in BDT shown in Fig. 3(b) differ greatly from those of the Au-atom-BDT-Au-atom molecule shown in Fig. 2 , which has an energy gap of $0.77 \mathrm{eV}$ with $\mathrm{HOMO}$ and LUMO bands dominated by S $3 p$ states similar to previous calculations for the BDT molecule. ${ }^{29}$ However, S $3 p$ band is greatly broadened when BDT is attached to the Au slabs and the energy gap disappears. The major features in the PDOSs of $\mathrm{S}, \mathrm{C}$, and $\mathrm{H}$ states at $V_{\text {bias }}=1.5 \mathrm{~V}$ are similar to those shown in Fig. 3(b) except for some fine details.

The PDOSs of Au $5 d$ bands for $V_{\text {bias }}=1.5 \mathrm{~V}$ are shown in Figs. 4(a) and 4(b), in which the zero energy is chosen as the local chemical potential at the center of the trilayer system. The leading edges of the major $5 d$ features of the $\mathrm{Au}$ atoms in slabs 1 and 2 with $\mu_{1}=+0.75 \mathrm{eV}$ and $\mu_{2}=$ $-0.75 \mathrm{eV}$ shift downward from $\mu_{1}$ and upward toward $\mu_{2}$, respectively, which indicates an electron transfer from slab 1 to slab 2 that lowers and raises local electrostatic potential in slabs 1 and 2, respectively, when a bias is applied. This effect causes the DOS at $\mu_{1}, D\left(\mu_{1}\right)$, in slab 1 to be different from the corresponding $D\left(\mu_{2}\right)$ in slab 2 in contrast to previous calculations, which usually assumed $D\left(\mu_{1}\right)=D\left(\mu_{2}\right)$.

\section{THE TRANSPORT PROPERTIES OF THE GOLD-SAM-GOLD TRILAYER SYSTEM}

Figures 5(a) and 5(b) show the current-voltage $(I-V)$ and differential conductance-voltage $(C-V)$ characteristics of the gold-SAM-gold trilayer system of up to $V_{\text {bias }}=1.5 \mathrm{~V}$. The calculated currents and differential conductance have the same order of magnitude as the experimental data of Reed et $a l .{ }^{1}$ The calculated current at $V_{\text {bias }}=1.5 \mathrm{~V}$ is $0.050 \mu \mathrm{A}$, which is about 1.7 times that of Reed et al. ${ }^{1}$ of $\sim 0.03 \mu \mathrm{A}$. In comparison, previous calculations obtained currents of $\sim 10,{ }^{33} \sim 10$ (for BDT on jellium surface), ${ }^{24} \sim 0.3$ (for BDTAu-atom on jellium surface), ${ }^{24} \sim 20,{ }^{12} \sim 10,{ }^{38}$ and $\sim 10 \mu \mathrm{A}$ (Ref. 39) at the same bias voltage. The calculated differential conductance at $V_{\text {bias }}=0$ is $0.0062 \mu \mathrm{S}$, which is three times that of Reed et al. ${ }^{1}$ of $\sim 0.002 \mu \mathrm{S}$. In comparison, previous calculations obtained $V_{\text {bias }}=0$ differential conductances of $5.0,{ }^{33} 3$ (for BDT on jellium surface), ${ }^{24} \sim 0$ (for BDT-Auatom on jellium surface) $,^{24} 4.8,{ }^{12} 12,,^{37} 7,^{38}$ and $6 \mu \mathrm{S} .^{39}$ Here, it is emphasized that these previous calculations, except for Ref. 24, considered a contact geometry, in which the $\mathrm{S}$ end of BDT is bonded with three Au atoms at the threefold site of a flat $\mathrm{Au}(111)$ surface. The present study suggests that in the molecular-junction sample fabricated by Reed et al. ${ }^{1}$ the $\mathrm{S}$ end of BDT is bonded with a single $\mathrm{Au}$ atom rather than with multi-Au atoms. Figure 5(b) shows that the first
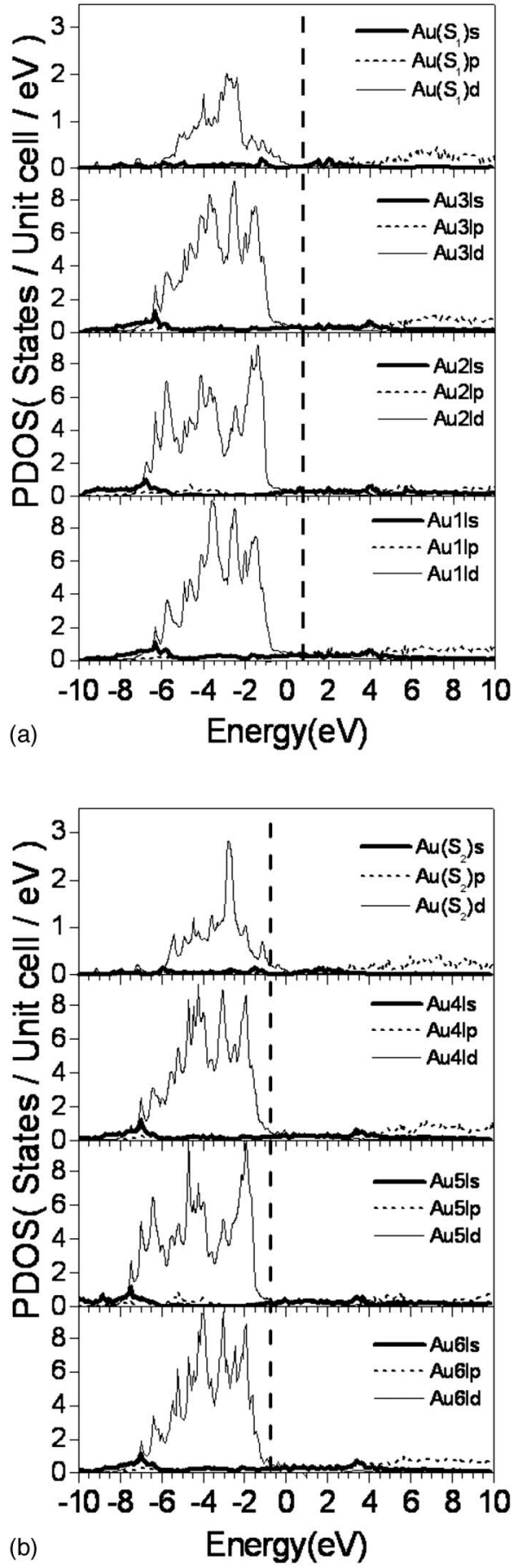

FIG. 4. PDOSs of Au atoms in (a) first to third layers and the one bonded with the $\mathrm{S}$ atom in slab $1, \mathrm{Au}\left(\mathrm{S}_{1}\right)$, and (b) fourth to sixth layers and the one bonded with the $\mathrm{S}$ atom in slab $2, \mathrm{Au}\left(\mathrm{S}_{2}\right)$. The vertical dash lines mark local chemical potentials.

peak in the $C$ - $V$ curve obtained in this study is located at $0.80 \mathrm{~V}$, which is smaller than the experimental value obtained by Reed et al. ${ }^{1}$ of $\sim 1.3 \mathrm{~V}$ by $0.5 \mathrm{~V}$. In comparison, the first peak in the $C$ - $V$ curve obtained by Di Ventra et $a l^{24}$ was $2.5 \mathrm{~V}$, which was larger than the experimental value by $1.2 \mathrm{~V}$.

The current calculations have also been done by treating $5 d$ electrons of the Au atoms in each slab, except for the three $\mathrm{Au}$ atoms coordinated with the single $\mathrm{Au}$ atom bonded with the $\mathrm{S}$ atom, as core electrons. In this case, only delocal- 


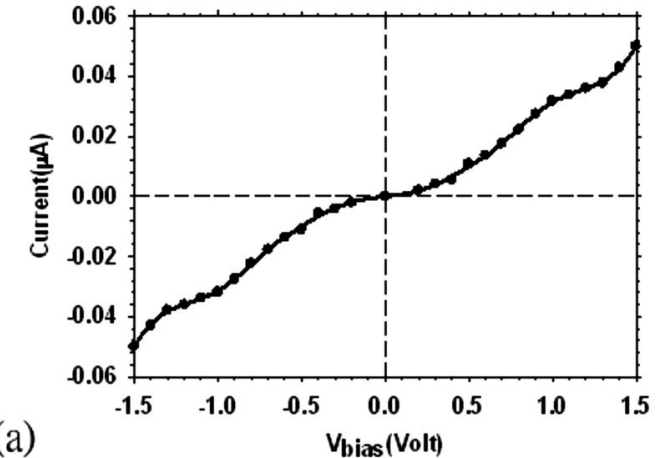

(a)

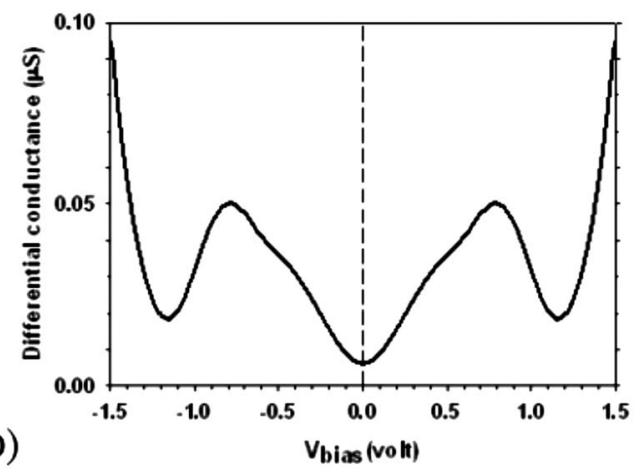

FIG. 5. (a) $I-V$ characteristic and (b) differential $C-V$ characteristic of the gold-SAM-gold trilayer system. In (a), the solid curve is obtained by fitting calculated values denoted by filled circles to seven-parameter rational functions. The circles in the negative bias voltage region are copied from those in the positive region by symmetry. The curve in (b) is obtained from the fitted functions.

ized Au $6 s p$ electrons participate in the bonding among $\mathrm{Au}$ atoms and contribute to current. The calculated $I-V$ characteristic is plotted in Fig. 6. This $I-V$ curve differs from that shown in Fig. 5(a) in both shape and magnitude. At $V_{\text {bias }}$ $=1.0 \mathrm{~V}$, the current is $0.0545 \mu \mathrm{A}$, which is 1.7 times larger than $0.0318 \mu \mathrm{A}$ when all $\mathrm{Au} 5 d$ electrons are treated as valence electrons. If $5 d$ electrons of all $\mathrm{Au}$ atoms are treated as core electrons, the current at $V_{\text {bias }}=1.0 \mathrm{~V}$ is overestimated further to become $0.087 \mu \mathrm{A}$, which is close to that obtained by Di Ventra et al. ${ }^{24}$ of $\sim 0.085 \mu \mathrm{A}$ when they considered

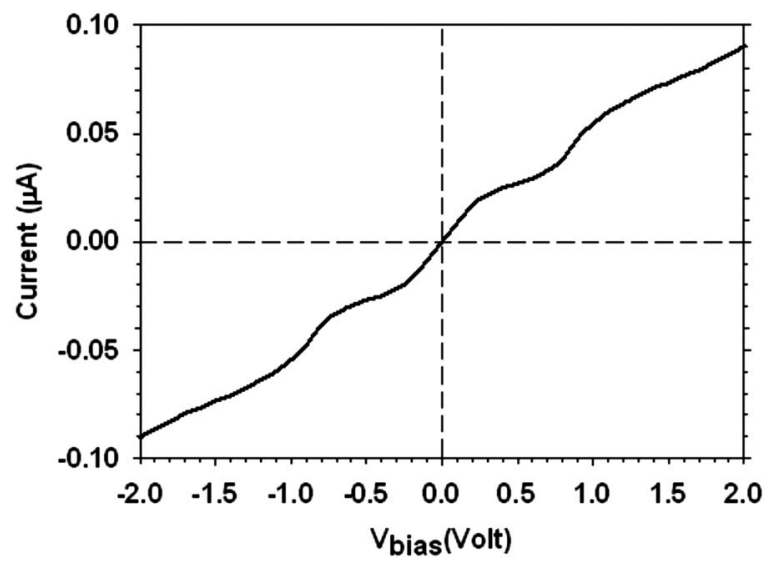

FIG. 6. $I-V$ characteristic of the gold-SAM-gold trilayer system when $5 d$ electrons of the Au atoms in the slabs, except for the three Au atoms coordinated with the single Au atom bonded with the $\mathrm{S}$ atom, are treated as core electrons. that the $\mathrm{S}$ end of BDT is bonded with a single Au atom, which is then attached to the flat jellium surface. The enhancement of current by confining Au $5 d$ electrons in the ion cores can be understood by the following reasons. First, it is stated previously that $\mathrm{Au} 5 d$ orbitals contribute substantially to the Au-S covalent bonding. The treatment of Au $5 d$ states as core states deprives hybridization between Au $5 d$ and $\mathrm{S} 3 p$ orbitals, which underestimates the $\mathrm{Au}-\mathrm{S}$ covalent bonding and consequently the potential barrier at the $\mathrm{Au}$ BDT contact. Second, when Au $5 d$ states are treated as valence states, part of Au $5 d$ states is delocalized so that the PDOS of $\mathrm{Au} 5 d$ states at $E_{F}$ is approximately equal to that of $6 s$ and $6 p$ states as stated previously. Let $D_{\mathrm{Au}}\left(E_{F}\right)$ be the total density of Au states at $E_{F}$, the PDOS of combined $6 s$ and $6 p$ states will be roughly $2 / 3 D_{\mathrm{Au}}\left(E_{F}\right)$. Assuming that the current is contributed dominantly by delocalized $6 s p$ states because they are much more itinerant than delocalized $5 d$ states, then $I \propto 2 / 3 D_{\mathrm{Au}}\left(E_{F}\right)$. If $1 / 3 D_{\mathrm{Au}}\left(E_{F}\right)$ of Au $5 d$ states are replaced by Au $6 s p$ states, then $I \propto D_{\mathrm{Au}}\left(E_{F}\right)$, i.e., the current will be enhanced by $\frac{3}{2}=1.5$ times, which is close to the calculated 1.7 times stated previously. This analysis suggests that the enhancement of the current is due to replacement of near $E_{F}$ delocalized Au $5 d$ states by much more itinerant delocalized $6 s p$ states. Figure 3(a) shows that PDOSs of Au $6 s$ and $6 p$ states in bulk gold crystal and the three-layer slab are not free-electron-like, which is proportional to the square root of the energy measured from the bottom of the $6 s p$ bands. The present result implies that the use of freeelectron-like electronic states, which are more $s p$ like than $d$ like and free-electron-like DOS to represent the electronic structure of the gold electrode may overestimate the current.

\section{CONCLUSION}

An integrated piecewise thermal equilibrium approach based on the first-principles calculation method has been developed to calculate $I-V$ and differential $C$ - $V$ characteristics of the gold-BDT-gold molecular junction. The calculated currents and differential conductance have the same order of magnitude as experimental ones. Much better quantitative agreement between calculated currents and experimental data of Reed et al. ${ }^{1}$ suggests that the $\mathrm{S}$ end of BDT is bonded with a single $\mathrm{Au}$ atom rather than with multi-Au atoms considered in most previous theoretical studies. This study also finds a bias induced electron transfer between the two electrodes, which causes the local electronic structures in the two electrodes to be different. When the $5 d$ electrons of all Au atoms are treated as core electrons, the current is overestimated, which can be understood as an underestimate of the Au-S covalent bonding and the potential barrier and the replacement of delocalized Au $5 d$ carriers by more itinerant and longer ranged delocalized $\mathrm{Au} 6 s p$ carriers in the electrodes.

\section{ACKNOWLEDGMENTS}

This work was supported by the National Science Council of Taiwan (Contract No. NSC 94-2112-M-110-012).

\footnotetext{
${ }^{1}$ M. A. Reed, C. Zhou, C. J. Müller, T. P. Burgin, and J. M. Tour, Science 278, 252 (1997).

${ }^{2}$ J. Chen, M. A. Reed, A. M. Rawlett, and J. M. Tour, Science 286, 1550
} 
(1999).

${ }^{3}$ W. Tian, S. Datta, S. Hong, R. Reifenberger, J. I. Henderson, and C. P Kubiak, J. Chem. Phys. 109, 2874 (1998).

${ }^{4}$ X. Xiao, B. Xu, and N. J. Tao, Nano Lett. 4, 267 (2004).

${ }^{5}$ T. Dadosh, Y. Gordin, R. Krahne, I. Khivrich, D. Mahalu, V. Frydman, J. Sperling, A. Yacoby, and I. Bar-Joseph, Nature (London) 436, 677 (2005).

${ }^{6}$ M. Kiguchi, S. Miura, K. Hara, M. Sawamura, and K. Murakoshi, Appl. Phys. Lett. 91, 053110 (2007); 89, 213104 (2006).

${ }^{7}$ M. P. Samanta, W. Tian, S. Datta, J. I. Henderson, and C. P. Kubiak, Phys. Rev. B 53, R7626 (1996).

${ }^{8}$ S. Datta, W. D. Tian, S. H. Hong, R. Reifenberger, J. I. Henderson, and C. P. Kubiak, Phys. Rev. Lett. 79, 2530 (1997).

${ }^{9}$ E. G. Emberly and G. Kirczenow, Phys. Rev. B 58, 10911 (1998).

${ }^{10}$ S. N. Yaliraki, M. Kemp, and M. A. Ratner, J. Am. Chem. Soc. 121, 3428 (1999).

${ }^{11}$ S. N. Yaliraki, A. E. Roitberg, C. Gonzalez, V. Mujica, and M. A. Ratner, J. Chem. Phys. 111, 6997 (1999).

${ }^{12}$ Y. Xue and M. A. Ratner, Phys. Rev. B 68, 115406 (2003).

${ }^{13}$ P. E. Kornilovitch and A. M. Bratkovsky, Phys. Rev. B 64, 195413 (2001)

${ }^{14}$ A. M. Bratkovsky and P. E. Kornilovitch, Phys. Rev. B 67, 115307 (2003)

${ }^{15}$ C.-K. Wang and Y. Luo, J. Chem. Phys. 119, 4923 (2003).

${ }^{16}$ C.-K. Wang, Y. Fu, and Y. Luo, Phys. Chem. Chem. Phys. 3, 5017 (2001).

${ }^{17}$ J. Nara, H. Kino, N. Kobayashi, M. Tsukada, and T. Ohno, Thin Solid Films 438-439, 221 (2003).

${ }^{18}$ A. Tikhonov, R. D. Coalson, and Y. Dahnovsky, J. Chem. Phys. 117, 567 (2002).

${ }^{19}$ K. Stokbro, J. Taylor, M. Brandbyge, J.-L. Mozos, and P. Ordejon, Comput. Mater. Sci. 27, 151 (2003).

${ }^{20}$ N. D. Lang, Phys. Rev. B 52, 5335 (1995).

${ }^{21}$ P. Delaney and M. Di Ventra, Appl. Phys. Lett. 75, 3787 (1999).

${ }^{22}$ M. Di Ventra, S. T. Pantelides, and N. D. Lang, Appl. Phys. Lett. 76, 3448 (2000).

${ }^{23}$ M. Di Ventra, S. G. Kim, S. T. Pantelides, and N. D. Lang, Phys. Rev. Lett. 86, 288 (2001).

${ }^{24}$ M. Di Ventra, S. T. Pantelides, and N. D. Lang, Phys. Rev. Lett. 84, 979 (2000).

${ }^{25}$ M. Di Ventra and N. D. Lang, Phys. Rev. B 65, 045402 (2001).

${ }^{26}$ M. Di Ventra, S. T. Pantelides, and N. D. Lang, Phys. Rev. Lett. 88, 046801 (2002).

${ }^{27}$ M. Di Ventra, N. D. Lang, and S. T. Pantelides, Chem. Phys. 281, 189 (2002).

${ }^{28}$ Y. Xue and M. A. Ratner, Phys. Rev. B 69, 085403 (2004).

${ }^{29}$ H. Kondo, H. Kino, J. Nara, T. Ozaki, and T. Ohno, Phys. Rev. B 73, 235323 (2006).

${ }^{30}$ H. Chen, J. Q. Lu, J. Wu, R. Note, H. Mizuseki, and Y. Kawazoe, Phys. Rev. B 67, 113408 (2003).

${ }^{31}$ D. Waldron, P. Haney, B. Larade, A. MacDonald, and H. Guo, Phys. Rev. Lett. 96, 166804 (2006).
${ }^{32}$ W. T. Geng, J. Nara, and T. Ohno, Appl. Phys. Lett. 85, 5992 (2004).

${ }^{33}$ S. V. Faleev, F. Léonard, D. A. Stewart, and M. van Schilfgaarde, Phys. Rev. B 71, 195422 (2005).

${ }^{34}$ E. G. Emberly and G. Kirczenow, Phys. Rev. B 64, 235412 (2001).

${ }^{35}$ J. C. Cuevas, J. Henrich, F. Pauly, W. Wenzel, and G. Schön, Nanotechnology 14, R29 (2003).

${ }^{36}$ R. Pati, L. Senapati, P. M. Ajayan, and S. K. Nayak, Phys. Rev. B 68, 100407(R) (2003).

${ }^{37}$ F. Evers, F. Weigend, and M. Koentopp, Phys. Rev. B 69, 235411 (2004).

${ }^{38}$ J. Tomfohr and O. F. Sankey, J. Chem. Phys. 120, 1542 (2004).

${ }^{39}$ P. S. Damle, A. W. Ghosh, and S. Datta, Phys. Rev. B 64, 201403 (2001).

${ }^{40}$ A. Nitzan and M. A. Ratner, Science 300, 1384 (2003).

${ }^{41}$ S. Datta, Electronic Transport in Mesoscopic Systems (Cambridge University Press, Cambridge, England, 1995), Chap. 2.

${ }^{42}$ J. Taylor, H. Guo, and J. Wang, Phys. Rev. B 63, 245407 (2001); 63, 121104 (2001)

${ }^{43}$ Y. Xue, S. Datta, and M. A. Ratner, Chem. Phys. 281, 151 (2002).

${ }^{44}$ M. Brandbyge, J.-L. Mozos, P. Ordejón, J. Taylor, and K. Stokbro, Phys. Rev. B 65, 165401 (2002)

${ }^{45}$ J. J. Palacios, A. J. Pérez-Jiménez, E. Louis, E. SanFabián, and J. A. Vergés, Phys. Rev. B 66, 035322 (2002).

${ }^{46}$ S.-H. Ke, H. U. Baranger, and W. Yang, Phys. Rev. B 70, 085410 (2004).

${ }^{47}$ O. F. Sankey and D. J. Niklewski, Phys. Rev. B 40, 3979 (1989).

${ }^{48}$ M.-H. Tsai, O. F. Sankey, and J. D. Dow, Phys. Rev. B 46, 10464 (1992).

${ }^{49}$ G. B. Bachelet, D. R. Hamnn, and M. Schlüter, Phys. Rev. B 26, 4199 (1982).

${ }^{50}$ R. W. Jansen and O. F. Sankey, Phys. Rev. B 36, 6520 (1987).

${ }^{51}$ D. M. Ceperley and G. J. Alder, Appl. Phys. Lett. 45, 566 (1980).

${ }^{52} \mathrm{H}$. Hellmann, Einfuhrung in die Quantumchemie (Franz Deutsche, Leipzig, 1937); R. P. Feynman, Phys. Rev. 56, 340 (1939); B. M. Deb, Rev. Mod. Phys. 45, 22 (1973).

${ }^{53}$ R. V. Kasowski, M.-H. Tsai, T. N. Rhodin, and D. D. Chambliss, Phys. Rev. B 34, 2656 (1986).

${ }^{54}$ In the modified PSF method, the projections of the tailing functions of the muffin-tin orbitals of surrounding atoms onto a given atom are calculated using two-dimensional structure factors. [K. Kambe, Z. Naturforsch. A 22A, 322 (1967a); 22A, 422 (1967b); 23A, 1280 (1968); J. B. Pendry, Low Energy Electron Diffraction (Academic, London, 1974), p. 137].

${ }^{55}$ O. K. Andersen, Phys. Rev. B 12, 3060 (1975).

${ }^{56}$ L. Hedin and B. I. Lundqvist, J. Phys. C 4, 2064 (1971).

${ }^{57}$ S. L. Cunningham, Phys. Rev. B 10, 4988 (1974).

${ }^{58}$ CRC Handbook of Chemistry and Physics, 80th ed., edited by D. R. Lide, (CRC, Boca Raton, FL, 1999-2000).

${ }^{59}$ Y. Yourdshahyan, H. K. Zhang, and A. M. Rappe, Phys. Rev. B 63, 081405(R) (2001).

${ }^{60}$ X.-G. Zhang, K. Varga, and S. T. Pantelides, Phys. Rev. B 76, 035108 (2007). 